\title{
Correction to: Hard X-Ray/Soft Gamma-Ray Experiments and Missions: Overview and Prospects
}

\author{
Erica Cavallari $^{1} \cdot$ Filippo Frontera $^{1,2}$
}

Published online: 26 June 2019

(C) Springer Nature B.V. 2019

\section{Correction to: Space Sci. Rev. (2017) 212: 429-518 \\ https://doi.org/10.1007/s11214-017-0426-9}

This article was published with erroneous information in the last statement of the first paragraph on page 448. Please find in this document the correct paragraph that should regarded as the final version by the reader.

The Konus results were outstanding. Concerning GRBs, we wish to mention the important discovery, within single GRBs, of a time-resolved correlation between luminosity and peak energy (interpreted as bremsstrahlung temperature) of the $E F(E)$ spectrum (Golenetskii et al. 1983), and the earliest evidence of an isotropic distribution of the GRB positions in the sky (Mazets et al. 1981; Mazets and Golenetskii 1988) (see Fig. 4). Other key results concern the discovery on 5 March 1979 of the first pulsating burst, later called Soft Gamma Ray Repeater (SGR), from the supernova remnant N49 in the Large Magellanic Cloud (Mazets et al. 1979b): SGR 0526-66. Konus detected a total of 16 recurrent bursts from this source (Golenetskii et al. 1984) and obtained the earliest detections of the famous SGR 1806-20 and SGR 1900+14 (Mazets et al. 1979a).

Publisher's Note Springer Nature remains neutral with regard to jurisdictional claims in published maps and institutional affiliations.

The online version of the original article can be found under https://doi.org/10.1007/s11214-017-0426-9.

F. Frontera

frontera@fe.infn.it

E. Cavallari

ericacavallari@gmail.com

1 Physics and Earth Sciences Department, University of Ferrara, Via Saragat, 1, 44122 Ferrara, Italy

2 National Institute of Astrophysics (INAF), Institute of Space Astrophysics and Cosmic Physics (IASF), Bologna, Italy 